Return to the Manage Active Submissions page at http://spie.org/submissions/tasks.aspx and approve or disapprove this submission. Your manuscript will not be published without this approval. Please contact author_help@spie.org with any questions or concerns.

\title{
Lab- and field-test results of MFIG, the first real-time vacuum-contamination sensor
}

\author{
Diederik Maas*, Pim Muilwijk, Michel van Putten, Frank de Graaf, \\ Olaf Kievit, Patrique Boerboom and Norbert Koster \\ TNO, Stieltjesweg 1, 2628CK Delft, The Netherlands
}

\begin{abstract}
To produce high-end semiconductor products, clean vacuum is often required. Even small amounts of high-mass molecules can reduce product yield. The challenge is to timely detect the presence of relevant contaminants. This is where MFIG can help. The mass-filtered ion gauge sensor (MFIG) continuously and selectively monitors the presence of high-mass contaminant molecules with a sensitivity down to $1 \mathrm{E}-13 \mathrm{mbar}$ at total pressures up to $1 \mathrm{E}-5 \mathrm{mbar}$.

This contribution presents laboratory and field-test data to demonstrate the capabilities of the latest version of the MFIG sensor in continuously and selectively detecting high-mass contaminant molecules in (U)HV vacuum.

Keywords: Molecular contamination; Vacuum; Sensor; Detection; Monitoring; Carbon contamination; Semiconductor manufacturing; Yield enhancement.
\end{abstract}

\section{INTRODUCTION}

To produce high-end semiconductor products, clean vacuum is often required. Even small amounts of high-mass molecules can reduce product yield. The challenge is to timely detect the presence of relevant contaminants. This is where MFIG can help [1]. The mass-filtered ion gauge sensor (MFIG) continuously and selectively monitors the presence of high-mass contaminant molecules with a sensitivity down to $1 \mathrm{E}-13 \mathrm{mbar}$ at total pressures up to $1 \mathrm{E}-5 \mathrm{mbar}$. This contribution presents laboratory and field-test data to demonstrate the capabilities of the latest version of the MFIG sensor in continuously and selectively detecting high-mass contaminant molecules in (U)HV vacuum. Many processing steps in semiconductor manufacturing require clean vacuum to reach a high yield. With shrinking device dimensions, even UHV is no longer always clean enough to get a constant high yield. Both vacuum system parts and samples are potential contaminant sources. Extremely low partial pressures (1E-15 - 1E-11 mbar) of contaminants can already kill yield [2,3]. The annual cost per 1\% yield loss is estimated at $100 \mathrm{M} \$$ per fab [4]. Such a high number justifies the development of new vacuum contamination sensors, such as e.g. MFIG.

TNO's development of MFIG started in 2007, when experts realized that IC manufacturing with EUV needs extremely clean vacuum, while existing sensors are either too slow, too expensive or too insensitive for real-time monitoring of vacuum quality. The promising results of a "quick-and-clean" test were well-received at the AVS fall meeting in 2008. Hence TNO incubated the concept in the ENIAC JU EEM450PR project, in which both instrumentation was improved and application requirements were clarified by interacting with the consortium partners. TNO was awarded an NanoNextNL valorization grant in 2015 to mature the instrumentation for better sensitivity and selectivity and cost, and to collect field test data to demonstrate MFIG's performance in realistic usage conditions. The field test was conducted at the Applied Materials PDC (Process Diagnostics and Control group) in Rehovot, Israel, in ENIAC JU project E450EDL.

This paper is organized as follows: the remainder of the introduction reviews the main drivers for vacuum contaminant monitoring in surface contamination. Section 2 outlines the sensor design and field test set-up. Section 3 presents lab and field test results, which are discussed in section 4. Section 5 summarizes conclusions and outlook.

\subsection{Surface contamination}

Surface contamination often occurs when a precursor and a highly energetic particle are simultaneously present at the surface. Figure 1 displays both an illustration, taken from [3], on the mechanisms involved, and a helium ion microscope

*diederik.maas@tno.nl; www.tno.nl 
Return to the Manage Active Submissions page at http://spie.org/submissions/tasks.aspx and approve or disapprove this submission. Your manuscript will not be published without this approval. Please contact author_help@spie.org with any questions or concerns.

image of a locally contaminated EUV reticle after SEM inspection, taken from [5]. In (U)HV systems, the contamination-precursor mix often comprises volatile (mass $<100 \mathrm{amu}$ ) and non-volatile (mass $>100 \mathrm{amu}$ ) hydrocarbons [3]. The heavier hydrocarbons reside considerably longer on a surface [6-8], and hence are much more effectively dissociated when irradiated by an EUV [2,3], or scanning electron beam [5]. To prevent and control surface contamination, it is necessary to quantify and monitor the partial pressure of non-volatile hydrocarbons [1].
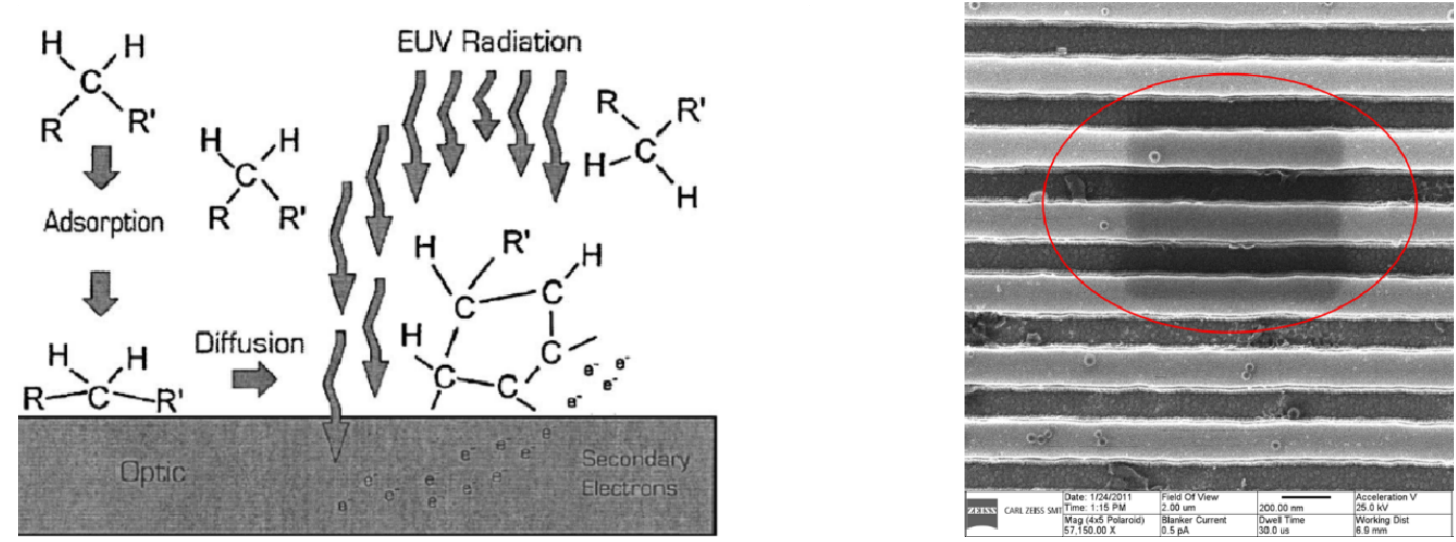

Figure 1 Application examples of surface contamination as induced by the dissociation of precursor molecules on the surface after irradiation by a high-energy beam: EUV (left), by courtesy of [3] or electron beam (right), by courtesy of [5]. The resulting carbon contamination on the surface causes a decrease of the reflectivity of an EUV multi-layer mirror [2,3] and can be easily visualized using a helium ion microscope [5].

\subsection{Contamination precursor monitoring: RGA vs. MFIG}

At present, the default vacuum contamination monitoring method deploys two sensors: an Ion Gauge (IG) to record the total pressure $P_{t o t}$ and a residual gas analyzer (RGA) to analyze the mass spectrum of the molecules in the vacuum. The RGA typically records a mass spectrum by the scanning of an AC quadrupole field while measuring the relative presence $R P_{m}$ of ions from e.g. $m=1 \mathrm{amu}$ (atomic hydrogen) up to an upper mass limit (UML) of typically $m=U M L=200 \mathrm{amu}$ (under the assumption that only singly-charged ions are present). Summation of the RGA signals $R P_{m}$ over the full mass range yields the total ion current, which is, when the vacuum is in steady state, by definition equal to the total pressure $P_{t o t}$ as measured by the IG. Hence, one arrives at partial pressures of all masses $P_{m}$ by calibrating the relative signals $R P_{m}$ using the IG signal. One can calculate the contamination precursor signal $C P$ [mbar] by summation of the calibrated RGA signals $P_{m}$ for masses above the use-case-specific lower mass limit $(L M L)$ up to the $U M L$ of the RGA;

$$
C P=\sum_{m=L M L}^{U M L} P_{m} .
$$

$C P$ is a measure for the vacuum cleanliness. The tool operator can use the reported $C P$ to decide if (preventive) maintenance is needed to maintain or improve the yield of the process. However, in practice the RGA-signal based $C P$ has some limitations. Firstly, the tuning of an RGA to yield reproducible results requires an expert operator. Secondly, an RGA monitors only one particular mass at a time. Hence at the required sensitivity level, a full mass range scan can take up to tens of minutes. Another consequence is the risk of missing short contaminating events when the RGA is de-tuned from the mass of that particular contaminant precursor. Ergo, to improve vacuum contamination control for the (semicon) industry new fast and simple sensors are needed that measure the integrated partial pressure for mass ranges of 100-200 amu with a high sensitivity and data rate. It is in response to this need that TNO developed the MFIG sensor to enable field tests in lab or pilot-line tools at potential OEM customer sites.

\section{EXPERIMENTAL}

\subsection{MFIG sensor design}

The MFIG sensor consists of an electron impact ion source, ion optics comprising a filter for mass-separation and a Faraday electrode to record the ion current that is representative for the integrated partial pressure of the non-volatile contaminant molecules. The MFIG ion optics design was optimized using the concept of practical brightness, as outlined 
Return to the Manage Active Submissions page at http://spie.org/submissions/tasks.aspx and approve or disapprove this submission. Your manuscript will not be published without this approval. Please contact author_help@spie.org with any questions or concerns.

in [9], aiming for maximum sensitivity for molecular ions above $100 \mathrm{amu}$. Furthermore, maximum selectivity was achieved by suppressing the lighter-molecular-ion signal than the lower-mass-limit (LML) by a proprietary mass-filter. The $L M L$ is use-case-specific and ranges between $L M L=70-100$ amu for most practical cases. MFIG's $L M L$ can be set by the vacuum-tool operator. Compared to the continuous-mass-scanning by an RGA when operated in contaminationmonitoring mode, MFIG statically integrates the ion current of all ions with a mass above $100 \mathrm{amu}$. Thus, MFIG trades in mass-information for a sensitivity increase. As a consequence, the requirements on the ion optics are more relaxed. This enables MFIG to sample a substantially larger volume of the vacuum system than a typical RGA can monitor. Hence, the $\mathrm{S} / \mathrm{N}$ of the ion current signal is sufficiently high to enable a direct recording of the signal by a Tek 6485 current meter at sampling frequencies up to several Hz. This enables real-time contamination monitoring for the first time. Furthermore, the absence of a secondary electron multiplier in the MFIG signal chain extends the operating pressure range up to $1 \mathrm{E}-5$ mbar. This is substantially higher than for a typical RGA when operating at its highest sensitivity setting [10]. The MFIG sensor has a CF40 flange to connect to the host vacuum system. Last but not least, the MFIG sensor was constructed from materials that are fully compliant with Ultra-Clean Vacuum requirements.

\subsection{Set-up for laboratory and field tests}

Laboratory tests were performed at TNO to verify the sensitivity and selectivity of MFIG for heavy ions. To this end, both a RGA, a regular ion gauge (IG) and MFIG were mounted on a UHV vessel. This vacuum set-up was also equipped with a valve system for the controlled injection of gases, with the option to dilute these gases with nitrogen gas. To represent the class of non-volatile contaminant molecules that have masses between 100 and $200 \mathrm{amu}$, xenon (mass $M_{X e}$ $=131 \mathrm{amu}$ ) was selected. The ion optics and mass-filter settings were optimized for sensitivity for Xe. To verify MFIG's selectivity, molecular nitrogen, argon and krypton, with ion masses $M_{N 2}=28, M_{A r}=40, M_{K r}=84$, respectively, were injected into the vacuum chamber. Measurement of the suppression factor of the MFIG signal for these lighter ions for different settings of the mass-filter quantified the selectivity of MFIG.

For the field test at Applied Materials's PDC in Rehovot, Israel, an IG, MFIG and RGA were mounted on the main chamber of a state-of-the-art defect review (DR) SEM, see Figure 2. A vacuum adapter piece mounted MFIG on a KF16 port on the DR-SEM main chamber. The resulting reduced pumping speed may have lowered MFIG sensitivity when compared to the lab test. The IG and RGA were also mounted on KF16 ports. The MFIG mass filter settings, as determined from the laboratory tests, were verified using krypton, which was introduced into the main chamber using a needle valve. In the test week, the DR-SEM was in use for typical DR jobs on $300 \mathrm{~mm}$ wafers, which entered the main vacuum chamber through a load lock. The connection of the load lock to the main chamber could be opened or closed with a gate valve $(\mathrm{GV})$. During the field test, the tool status (wafer in/out, GV open/closed) and the IG, RGA and MFIG signals were continuously recorded.

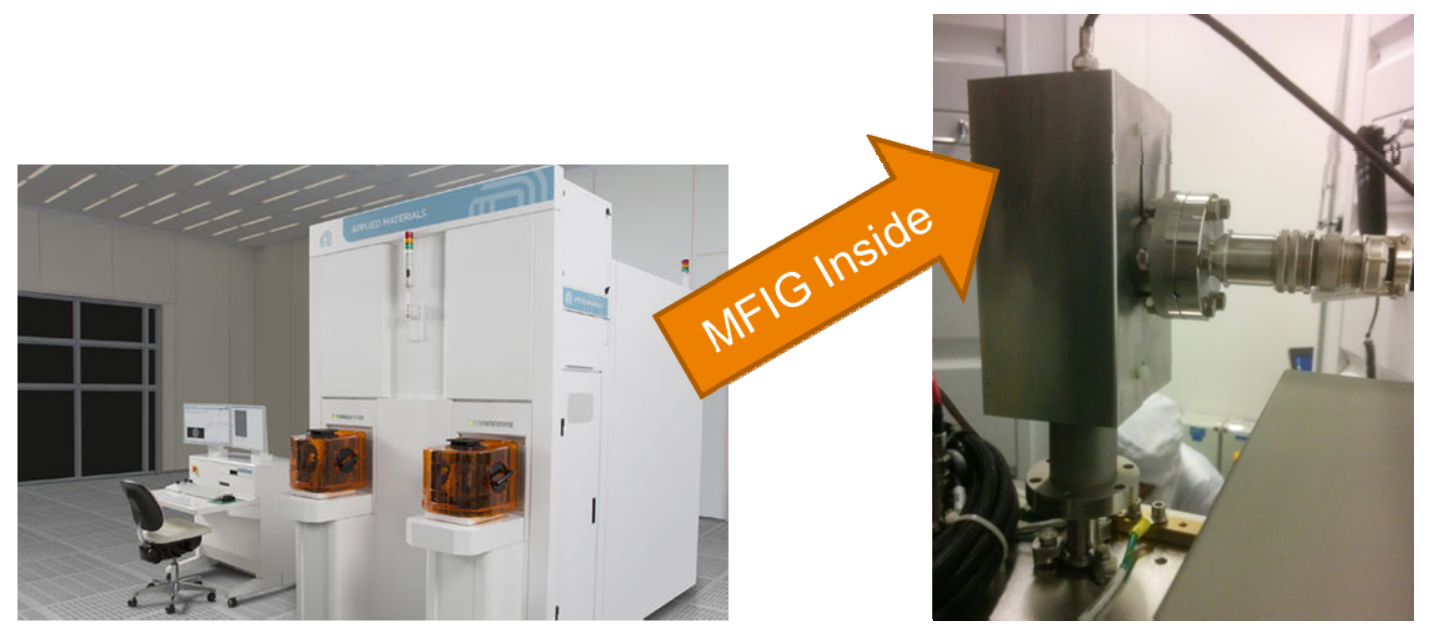

Figure 2 MFIG (right panel) as mounted on a state-of-the-art DR-SEM (left panel) for the field test at AMILs PDC.

\section{RESULTS}

In this section first the results of the lab test of MFIG at TNO are presented. Then we turn to data obtained during the field-test of the calibrated MFIG sensor at a state-of-the-art DR-SEM at AMILs PDC. 


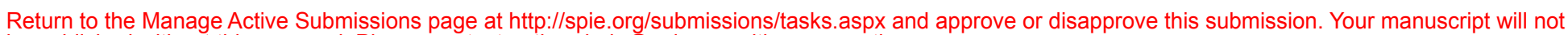
be published without this approval. Please contact author_help@spie.org with any questions or concerns.

\subsection{Lab test results on MFIG sensitivity and selectivity}

Figure 3 displays the MFIG response to the partial pressure of Xenon. The MFIG (and RGA) signals for Xe can be converted to mBar using the IG. MFIG's sensitivity for Xe as estimated based on the data in Figure 3, is $1.7 \mathrm{~A} / \mathrm{bar}$ (red squares) or at $6.8 \mathrm{~A} / \mathrm{bar}$ (blue squares). The relatively large discrepancy in the sensitivity estimate is attributed to either the uncertainty in the $\mathrm{Xe} / \mathrm{N}_{2}$ dilution factor or to too limited suppression of the $\mathrm{N}_{2}$ signal by the mass- filter, see e.g. Figure 4. The lower detection limit of MFIG $\left(L D L_{M F I G}\right)$ is set by the noise floor on the current meter read-out. From Figure 3, the MFIG electronic noise floor is estimated at $0.3 \mathrm{pA}$. Depending on which calibration data set (red vs. blue squares) is taken, the LDL ranges between $L D L_{M F I G-r e d} \cong 2 \mathrm{E}-10$ mbar and $L D L_{M F I G-b l u e} \cong 5 \mathrm{E}-11$ mbar.

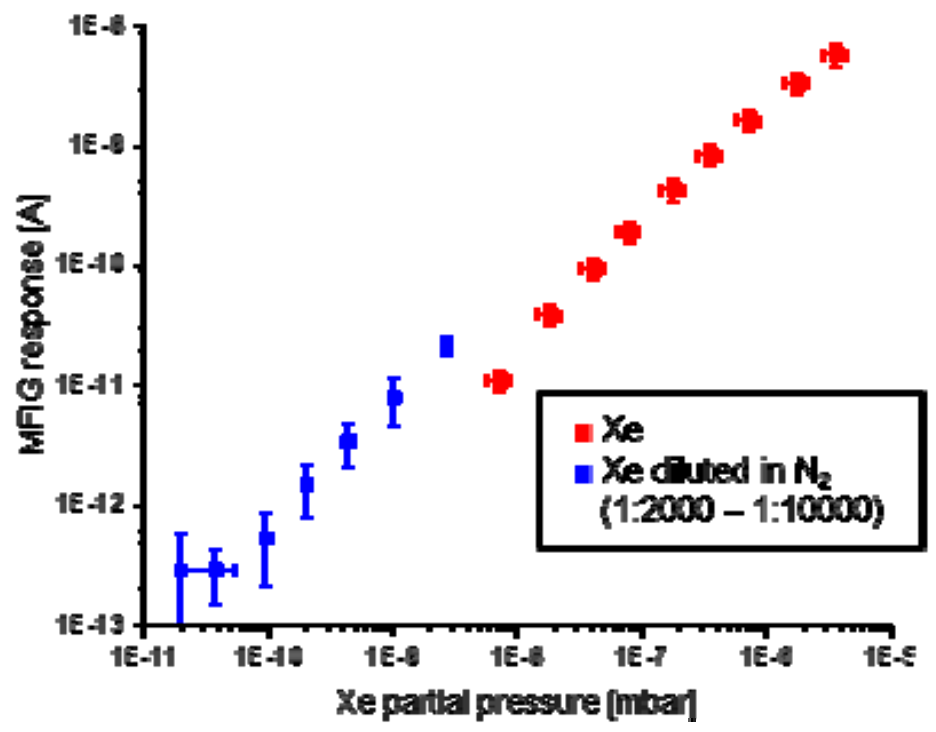

Figure 3 MFIG response to Xe partial pressure as measured with the RGA. The total pressure in the UHV system varied due to presence of the dilution gas $\mathrm{N}_{2}$. Note that MFIG operates over at least six orders of Xenon pressure variation, at total pressures up to $1 \mathrm{E}-5$ mbar.

Figure 4 displays the transmission of MFIG as measured using controlled injection of $\mathrm{N}_{2}, \mathrm{Ar}, \mathrm{Kr}$ and $\mathrm{Xe}$ into the UHV chamber for a mass-filter setting with maximal sensitivity for Xenon $\left(\mathrm{T}_{\mathrm{Xe}} \cong 0.9\right)$. For each of these gasses, the MFIG sensitivity $[\mathrm{A} / \mathrm{mbar}]$ was measured with the mass-filter ON and OFF over a pressure range of at least four orders. The MFIG transmission factor is the ratio of the MFIG sensitivity with the mass filter ON over that with the mass filter OFF.

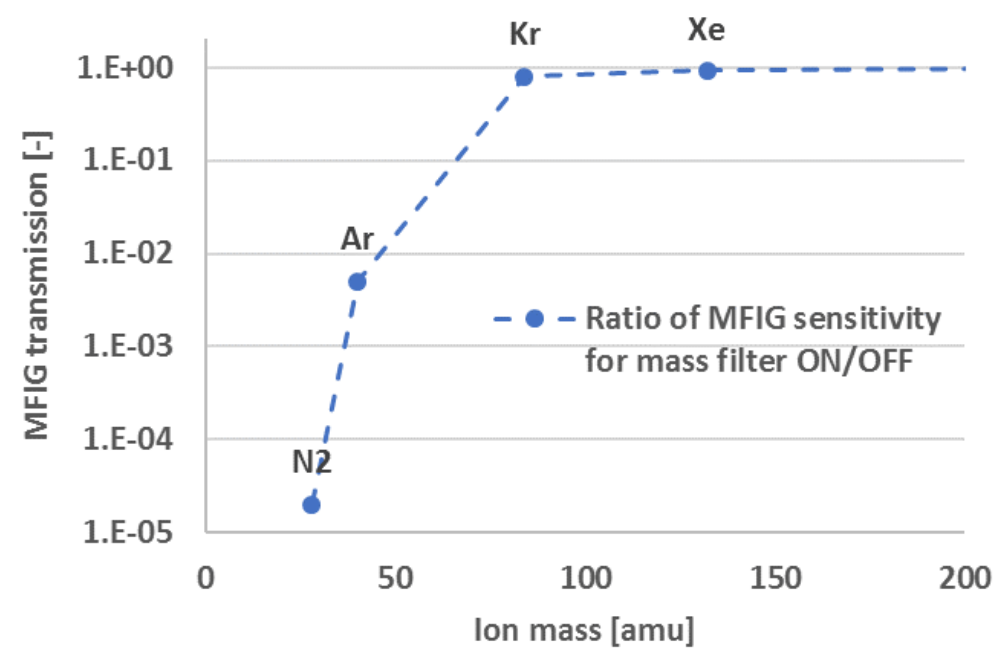

Figure 4 Lab test results of the MFIG selectivity, defined as the mass-filter transmission for ions at a particular mass. The dashed line is only a guide-to-the-eye. 


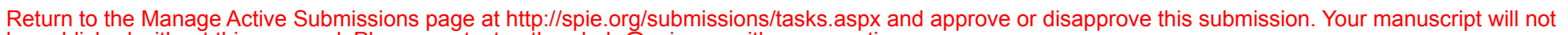
be published without this approval. Please contact author_help@spie.org with any questions or concerns.

\subsection{Field test results for MFIG and an RGA on a state-of-the-art DR-SEM}

Figure 5 shows time traces that report on tool status and IG and MFIG sensor readings, as recorded during operation of a DR-SEM in AMIL's PDC. Both the IG and MFIG signal rise instantaneously when the gate valve between the load lock and main chamber opens to load a new wafer, not when unloading a reviewed wafer. After a GV-triggered pressure rise, the IG signal decays much faster than the MFIG signal, in agreement with the notion that the lighter and more volatile molecules are pumped out faster than the heavier non-volatile molecules. Furthermore, the MFIG signal shows numerous short-yet-significant incidents in the 10-31 minute time slot, which are invisible on the IG.
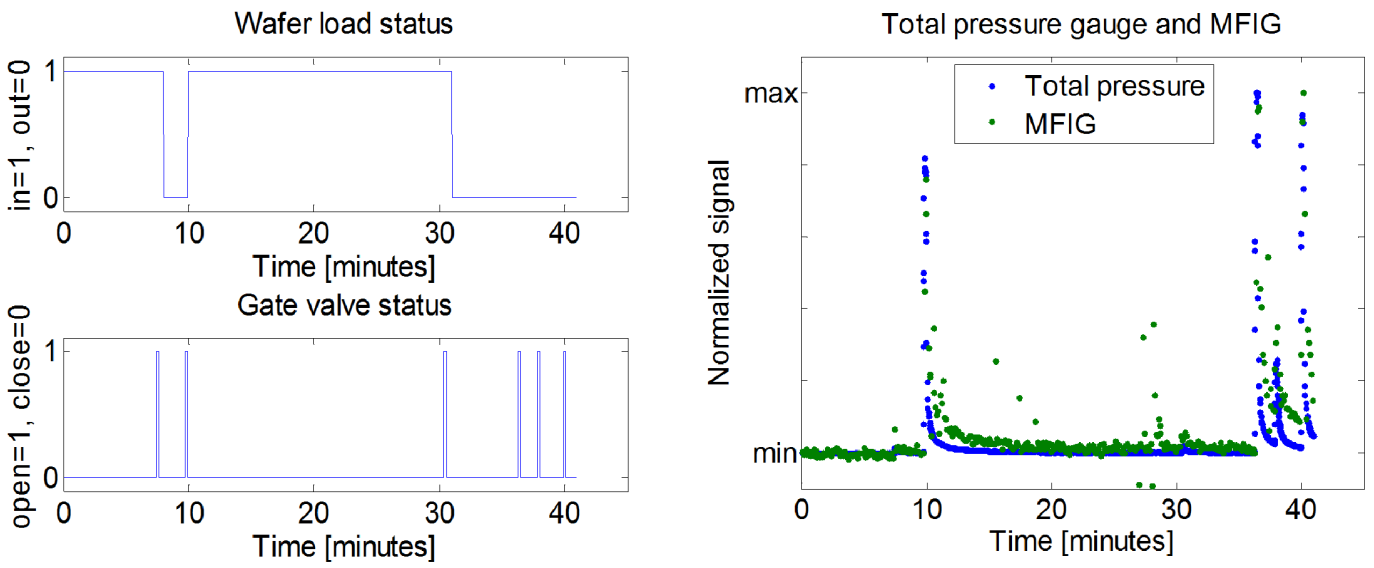

Figure 5 Time traces of the wafer load status of the chamber (top left), the valve status of the gate between load lock and main vacuum chamber (bottom left), and normalized ion pressure gauge (IG) and MFIG signals (right panel). The MFIG signal shows that heavier molecules pump out relatively slow, and that some short (!) actions in the SEM chamber cause desorption and hence transport of heavier molecules, potentially causing contamination.

Figure 6 shows similar time traces, benchmarking the MFIG signal to mass-integrated RGA signals for medium (mass range 50-100 amu) and heavy (mass range 100-200 amu) molecules. The MFIG signal obviously offers a better time resolution. Furthermore, the MFIG signal correlates well with both RGA signals, as was to be expected when taking the MFIG transmission for ions in these mass ranges into account (see Figure 4). Moreover, MFIG also provides timestamps to actions that cause the rise of both RGA signals. This provides SEM instrument and application developers with unique clues on how to improve their contamination control.
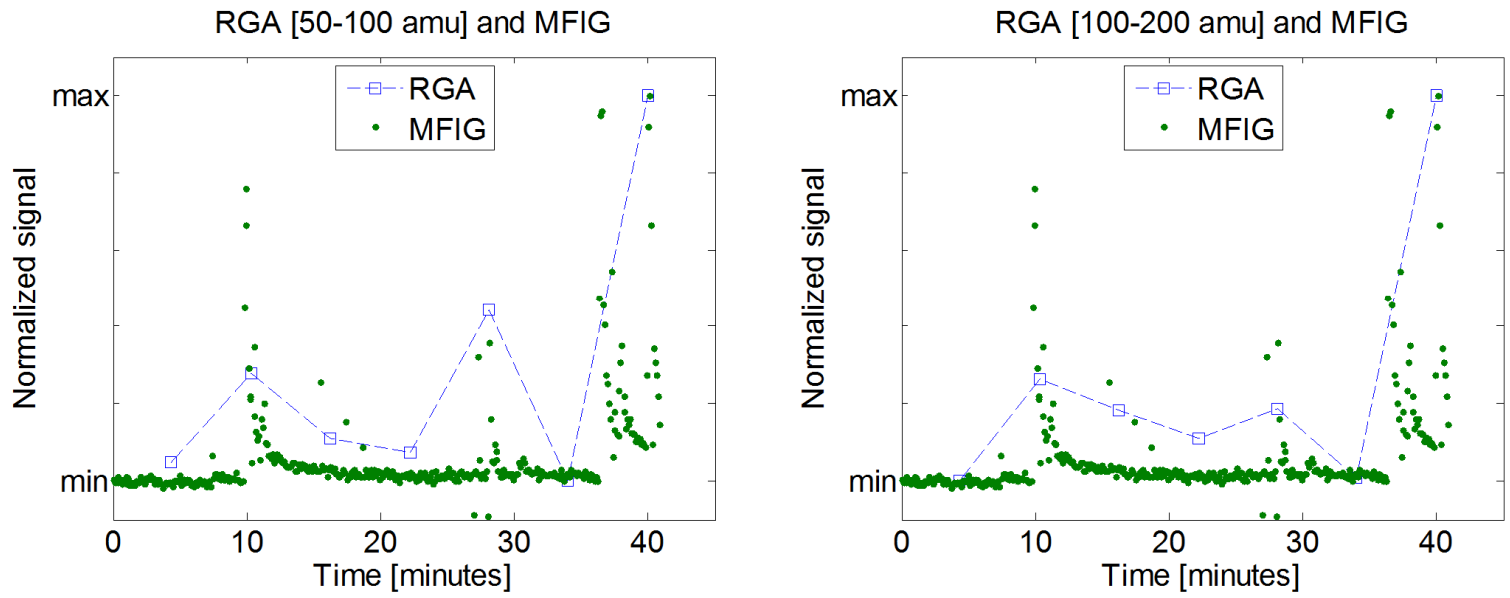

Figure 6 Time traces of the MFIG signal (both panels) and mass-integrated RGA signals for volatile and non-volatile molecules (left and right panel, respectively). 
Return to the Manage Active Submissions page at http://spie.org/submissions/tasks.aspx and approve or disapprove this submission. Your manuscript will not be published without this approval. Please contact author_help@spie.org with any questions or concerns.

\section{DISCUSSION}

The laboratory tests have shown convincingly that the MFIG prototype performs well on sensitivity for non-volatile molecules, at a reasonable selectivity. In a next experiment, either some sensitivity for non-volatile molecules could be traded in for a higher selectivity, or the mass filter design could be further improved to transmit even less lighter ions. Either measure would improve the pressure dynamic range of the sensor, thus allowing for even better detection of ultralow amounts of non-volatile molecules at higher total operating pressures.

The field test of the MFIG sensor showed that MFIG is at least as sensitive as the RGA and as fast as the IG. This yields new and unique information on the dynamics of non-volatile molecules as may be triggered by transient events in the main chamber of the host vacuum system. Take for instance the short incidents as registered by MFIG and no other sensor. Such incidents can perhaps be correlated to specific actions in the main chamber, which may provide clues to tool developers to further improve the vacuum cleanliness. For instance, as these incidents are much shorter than the GVtriggered events, it is likely that they are caused by other fast and transient actions. These incidents decay much quicker than after any GV-action, indicating that the involved desorbed molecules are quickly recaptured, e.g. at the surfaces in the main chamber, which apparently act like a getter pump for non-volatile molecules.

\section{CONCLUSIONS AND OUTLOOK}

Non-volatile heavy hydrocarbons in vacuum systems can negatively impact yield in semiconductor manufacturing, even at extremely low partial pressures. Currently, no commercial sensor can detect these non-volatile molecules at the required sensitivity and selectivity level real-time. This paper shows that MFIG can monitor the presence of these contaminants real-time (i.e. with sampling frequencies between $0.1-10 \mathrm{~Hz}$ ) under regular vacuum process conditions at the required sensitivity, and almost sufficient selectivity. Furthermore, the field test of the MFIG sensor at a state-of-theart DR-SEM yielded new data as well as insights on the contamination precursor dynamics in SEM.

TNO aims to further develop MFIG with application-development and industrialization partners to create impact in yield improvement in semiconductor manufacturing.

\section{ACKNOWLEDGEMENTS}

The research described in this paper was performed in the framework of the NanoNextNL Valorisation Programme, and partly linked to the ENIAC EEM450PR and ECSEL E450EDL projects. TNO gratefully acknowledges funding by NanoNextNL, the ENIAC and ECSEL Joint Undertaking and the Netherlands Enterprise Agency (RVO). Furthermore, we thank our TNO colleagues Evert Nieuwkoop and Johan Lugtenburg for the design and realization of the MFIG electronics. Last, but not least, we thank Irit Ruach-Nir, Yigal Dafna, Kfir Luria and Guy Eytan for sharing the RGA data and their hospitality during the field test at AMILs PDC in Rehovot, Israel.

\section{REFERENCES}

[1] Koster, N.B., "Ion gauge, a monitoring system and a method for determining a total integrated concentration of substances having a specific molecular weight in a gas sample," U.S. Patent 8749245B2 (2009).

[2] Mertens, B., et al. "Progress in EUV optics lifetime expectations," Microelectron. Eng. 73-74 (2004) 16.

[3] Hollenshead, J., and Klebanoff, L. "Modeling radiation-induced carbon contamination of extreme ultraviolet optics,” J. Vac. Sci. Techn. B 24 (2006) 64.

[4] Cheng, F.-T., Chang, J.Y.-C., Huang, H.-C., Kao, C.-A., Chen, Y.-L. and Peng, J.-L., "Benefit model of virtual metrology and integrating AVM into MES," IEEE Trans. Semicond. Manuf. 24 (2011) 261.

[5] Koster, N.B., Molkenboer, F. T., van Veldhoven, E., Oostrom, S., "Towards defect free EUVL reticles: Carbon and particle removal by single dry cleaning process, and pattern repair by HIM," Proc. SPIE 7969 (2011) 79690X.

[6] Wolkow, R.A., "Controlled molecular adsorption on silicon: Laying a foundation for molecular devices," Annu. Rev. Phys. Chem. 50 (1999) 413. 
Return to the Manage Active Submissions page at http://spie.org/submissions/tasks.aspx and approve or disapprove this submission. Your manuscript will not be published without this approval. Please contact author_help@spie.org with any questions or concerns.

[7] Gellman, A.J., Paserba, K.R., "Kinetics and mechanism of oligomer desorption from surfaces: n-alkanes on graphite," J. Phys. Chem. B 106 (2002) 13231.

[8] Ulbricht, H., Zacharia, R,. Cindir, N., Hertel, T., "Thermal desorption of gases and solvents from graphite and carbon nanotube surfaces," Carbon 44 (2006) 2931.

[9] Bronsgeest., M.S., Barth, J.E., Swanson, L.W. and Kruit, P., "Probe current, probe size, and the practical brightness for probe forming systems," J. Vac. Sci. Technol. B 26 (2008) 949.

[10] Dawson, P.H., "The quadrupole: System design and residual gas analyzer performance," J. Vac. Sci. Techn. A 4 (1986) 1709. 